\title{
EQUIVALENCE OF NEARBY DIFFERENTIABLE ACTIONS OF A COMPACT GROUP
}

\author{
BY RICHARD S. PALAIS ${ }^{1}$ \\ Communicated by Deane Montgomery, April 5, 1961
}

In this note we will be concerned with the proof and consequences of the following fact: if $\phi_{0}$ is a differentiable action of a compact Lie group on a compact differentiable manifold $M$, then any differentiable action of $G$ on $M$ sufficiently close to $\phi_{0}$ in the $C^{1}$-topology is equivalent to $\phi_{0}$.

1. Notation. In what follows differentiable means class $\mathrm{C}^{\infty}$. If $M$ and $V$ are differentiable manifolds, $\mathscr{M}(M, V)$ is the space of differentiable maps of $M$ into $V$ in the $C^{K}$-topology where $K$ is a positive integer or $\infty$ fixed throughout. We denote by Diff $(M)$ the group of automorphisms of $M$ topologized as a subspace of $\mathfrak{T}(M, M)$. As such it is a topological group. $D(M)$ is the subgroup of Diff $(M)$ consisting of diffeomorphisms which are the identity outside of some compact set and $D_{0}(M)$ is the arc component of $i_{M}$, the identity map of $M$, in $D(M)$. If $M$ is compact $D(M)$ is locally arcwise connected and $D_{0}(M)$ is open in $D(M)$ and in fact in $\mathfrak{T}(M, M)$. For a definition of the $C^{K}$-topology and a proof of the statements made above, see [6]. If $G$ is a Lie group we denote by $\mathfrak{Q}(G, M)$ the space of differentiable actions of $G$ on $M$, i.e. continuous homomorphisms of $G$ into Diff $(M)$, topologized with the compact-open topology. If $\phi: g \rightarrow g^{\phi}$ is an element of $a(G, M)$ then by a theorem of D. Montgomery [2] $\tilde{\phi}:(g, m) \rightarrow g^{\phi} m$ is an element of $\mathfrak{N}(G \times M, M)$. Given $\phi \in Q(G, M)$ and $f \in \operatorname{Diff}(M)$ then $\phi$ composed with the inner automorphism of Diff $(M)$ defined by $f$ is another element $f \phi$ of $a(G, M)\left(g^{f \phi}=f g^{\phi} f^{-1}\right)$. Clearly $(f, \phi) \rightarrow f \phi$ is jointly continuous ${ }^{2}$ and defines an action of Diff $(M)$ on $Q(G, M)$. We henceforth consider $\mathfrak{Q}(G, M)$ as a Diff $(M)$-space and, a fortiori as a $D(M)$ and $D_{0}(M)$ space. Note that the orbit space $Q(G, M) / \operatorname{Diff}(M)$ is just the set of equivalence classes of actions of $G$ on $M$.

2. Statement of main theorem and consequences. The following theorem will be proved in $\$ 3$.

Theorem A. If $M$ is a compact differentiable manifold and $G$ is a compact Lie group then the $\mathfrak{D}_{0}(M)$-space $a(G, M)$ admits local cross sections; i.e. given $\phi_{0} \in \mathbb{Q}(G, M)$ there is a neighborhood $U$ of $\phi_{0}$ in

\footnotetext{
1 Partially supported by NSF Grant No. G-14227.

2 This follows from the proposition in $[6, \S 1]$.
} 
$Q(G, M)$ and a continuous map $\chi: U \rightarrow D_{0}(M)$ such that $\chi\left(\phi_{0}\right)=i_{M}$ and $\chi(\phi) \phi_{0}=\phi$.

Corollary 1. If $\phi_{t}$ is a continuous arc in $a(G, M)$ then there is a continuous arc $f_{t}$ in $D_{0}(M)$ such that $f_{0}=i_{M}$ and $\phi_{t}=f_{t} \phi_{0}$.

Remarks. Corollary 1 was proved in [7] by the author and T. E. Stewart under the added hypothesis that $(g, m, t) \rightarrow \tilde{\phi}_{t}(g, m)$ was jointly differentiable in all three variables. It was shown there by counter-example that Corollary 1 is invalid if we consider continuous rather than differentiable actions or if we drop either of the conditions that $G$ or $M$ be compact. It follows that all these conditions are also necessary for the validity of Theorem A.

Using that $D_{0}(M)$ is locally arcwise connected:

Corollary 2. $Q(G, M)$ is locally arcwise connected. If $\phi_{0} \in \mathfrak{Q}(G, M)$ then its orbit under $D_{0}(M)$ is its arc component in $Q(G, M)$ hence an open set, and its orbit under $\mathscr{D}(M)$ (i.e. the class of actions equivalent to $\left.\phi_{0}\right)$ is also open and so a union of arc components. Moreover if $\Delta=\left\{f \in D(M) \mid f \phi_{0}=\phi_{0}\right\}$ is the group of automorphisms of the differentiable $G$-space $\left(M, \phi_{0}\right)$ then $f \Delta \rightarrow f \phi_{0}$ is a homeomorphism of $D(M) / \Delta$ onto $D(M) \phi_{0}$.

Since $a(G, M)$ is separable metric and each equivalence class is open:

COROLlaRy 3. There are at most countably many inequivalent differentiable actions of $G$ on $M$.

REMARKs. It seems likely that by modifying a construction of R. Bing [1] one could construct uncountably many continuous actions of $Z_{2}$ on $S^{3}$ with fixed point sets pairwise inequivalently embedded 2-spheres. These actions would of course all be inequivalent.

The following extension theorem generalizes Theorem A. On the other hand it is an easy consequence of Theorem A above and Theorem $B$ of [6].

Theorem B. Let $H$ be a Lie group, $W$ a differentiable manifold (neither necessarily compact), $G$ a compact subgroup of $H$, and $M a$ compact submanifold of $W$. Let $\psi_{0} \in a(H, W)$ such that $M$ is invariant under $\psi_{0} \mid G$ and let $\phi_{0} \in Q(G, M)$ be the induced action of $G$ on $M$. Then given any neighborhood $\mathcal{O}$ of $M$ in $W$ there exists a neighborhood $U$ of $\phi_{0}$ in $a(G, M)$ and a map $\psi: U \rightarrow \mathfrak{Q}(H, W)$ such that $\psi\left(\phi_{0}\right)=\psi_{0}$, $\psi(\phi) \mid G$ leaves $M$ invariant and induces $\phi$ on $M$, and $\psi(\phi)$ agrees with $\psi_{0}$ outside $\mathcal{O}$. In fact there is a continuous map $\chi: U \rightarrow D_{0}(W)$ such that $\chi(\phi)$ is the identity outside $\mathcal{O}$ and such that $\psi(\phi)=\chi(\phi) \psi_{0}$ satisfies the above conditions. 
3. Proof of Theorem A. By a theorem proved independently by the author [5] and G. D. Mostow [4] there exists an orthogonal representation $g \rightarrow g^{\psi}$ of $G$ in a Euclidean vector space $V$ and a differentiable $\phi_{0}$-equivariant embedding $f_{0}: M \rightarrow V$. Let $\mathcal{O}$ be a tubular neighborhood of $f_{0}(M)$ in $V$ with respect to the Euclidean metric. Then $\mathcal{\theta}$ is invariant under the representation $\psi$ and the map $\pi: \mathcal{O} \rightarrow f_{0}(M)$ carrying a point of $\mathcal{O}$ into the unique nearest point of $f_{0}(M)$ is a differentiable equivariant retraction of $\mathcal{O}$ onto $f_{0}(M)$. Given $\phi \in Q(G, M)$ define $f_{\phi}: M \rightarrow V$ by $f_{\phi}(m)=\int g^{-1 \psi} f_{0}\left(g^{\phi} m\right) d g$ where the integral is with respect to Haar measure on $G$. Then (cf. [4, p. 434]) $f_{\phi}$ is $\phi$-equivariant and clearly $f_{\phi_{0}}=f_{0}$. The map $F_{\phi} \in \operatorname{mi}(G \times M, V)$ defined by $F_{\phi}(g, m)=\tilde{\psi}\left(g^{-1}, f_{0} \circ \tilde{\phi}(g, m)\right)$ is easily seen ${ }^{2}$ to depend continuously on $\phi \in Q(G, M)$ and since $f_{\phi}=\int F_{\phi}(g, m) d g$ it follows that $\phi \rightarrow f_{\phi}$ is a continuous map of $Q(G, M)$ into $\mathfrak{T}(M, V)$. Then for $\phi$ in a neighborhood $U^{\prime}$ of $\phi_{0}$ in $a(G, M) f_{\phi}(M) \subseteq \mathcal{O}$ so $\sigma(\phi)=f_{\phi_{0}}^{-1} \circ \pi \circ f_{\phi}$ $\in \mathfrak{M}(M, M)$. Now $\sigma: U^{\prime} \rightarrow \mathfrak{M}(M, M)$ is continuous ${ }^{2}$ and clearly $\sigma\left(\phi_{0}\right)=i_{M}$. Since $\mathscr{D}_{0}(M)$ is open in $\mathfrak{M}(M, M)$, for some smaller neighborhood $U$ of $\phi_{0}$ in $Q(G, M) \sigma: U \rightarrow D_{0}(M)$. Since $f_{\phi}, \pi$, at $f_{\phi_{0}}$ are respectively $\phi$-, $\pi$ - and $\phi_{0}$-equivariant maps into $(V, \psi)$ it follows that $\sigma(\phi) g^{\phi}=g^{\phi_{0}} \sigma(\phi)$ or putting $\chi(\phi)=\sigma(\phi)^{-1}, \chi(\phi) \phi_{0}=\phi$. Q.E.D.

4. Conjugacy of neighboring compact subgroups of $\operatorname{Diff}(M)$. It is suggested by Theorem A that an analogue of the Montgomery and Zippin conjugacy theorem for neighboring compact subgroups of a Lie group [3] might hold for Diff $(M)$, i.e. that given a compact subgroup $G$ of Diff $(M)$ every compact subgroup of Diff $(M)$ sufficiently close to $G$ is conjugate in Diff $(M)$ to a subgroup of $G$. This in fact is the case and was the basis of an earlier more complicated proof of Theorem A. A proof will appear elsewhere.

\section{REFERENCES}

1. R. Bing, A homeomorphism between the 3-sphere and the sum of two solid horned spheres, Ann. of Math. vol. 56 (1952) pp. 354-362.

2. D. Montgomery, Topological groups of differentiable transformations, Ann. of Math. vol. 46 (1945) pp. 382-387.

3. D. Montgomery and L. Zippin, A theorem on Lie groups, Bull. Amer. Math. Soc. vol. 48 (1942) pp. 448-452.

4. G. D. Mostow, Equivariant embeddings in Euclidean space, Ann. of Math. vol. 65 (1957) pp. 432-446.

5. R. S. Palais, Imbedding of compact, differentiable, transformation groups in orthogonal representations, J. Math. Mech. vol. 6 (1957) pp. 673-678.

6. - Local triviality of the restriction map for embeddings, Comment. Math. Helv. vol. 34 (1960) pp. 305-312.

7. R. S. Palais and T. E. Stewart, Deformations of compact differentiable transformation groups, Amer. J. Math. vol. 82 (1960) pp. 935-937.

BRANDEIS UNIVERSITY 